\title{
Utilization of Soft Computing for Risk Assessment of a Tunneling Project Using Geological Units
}

\author{
Sina Shaffiee Haghshenas ${ }^{a^{*}}$, Sami Shaffiee Haghshenas ${ }^{b}$, Milad Barmal ${ }^{c}$, \\ Niloofar Farzan ${ }^{\mathrm{c}}$ \\ ${ }^{a}$ Young Researchers and Elite Club, Rasht Branch, Islamic Azad University, Rasht, Gilan, Iran \\ ${ }^{b}$ Department of Civil Engineering, Astara Branch, Islamic Azad University, Astara, Gilan, Iran \\ ${ }^{c}$ BSc Graduate of Civil Engineering, University of Guilan, Gilan, Iran
}

Received 16 June 2016; Accepted 26 July 2016

\begin{abstract}
Soft computing is one of the most efficient tools for analysing risk taking in civil engineering projects. Therefore, in this paper, using Fuzzy C-means (FCM) technique as one of the most efficient and important classification methods in the area of soft computing, risk in the tunnelling project was evaluated and analysed. For this reason, considering three mechanical and physical parameters influencing the design and execution of the tunnelling project including overburden $(\mathrm{H})$, internal friction angle $(\varphi)$ and cohesion $(\mathrm{C})$, geological units were classified along the project's route. The present study has been conducted on the third section of Ghomrud tunnel as one of the greatest tunnelling projects in the centre of Iran. Results obtained from the evaluation of geological units along the tunnelling project's route after the validation of drilling rate index's results show the appropriate evaluation of the project's risk through fuzzy clustering technique.
\end{abstract}

Keywords: Soft Computing; Risk Analysis; Tunneling Project; Fuzzy C-Means Technique; Drilling Rate Index (DRI); Geological Units.

\section{Introduction}

Nowadays, with an increasing growth in the global science and technology, dealing with more complex and vague problems is inevitable. Therefore, the need for methods which understand complex and ambiguous problems is felt more than before. Neural networks, meta-heuristic methods, fuzzy neural networks and fuzzy logic are techniques of soft computing which have various applications in optimizing and solving problems in different sciences [1-5]. Several optimization approaches were used by Yagiz \& Karahan (2015) for estimating tunnel boring machine (TBM) performance [6], including Differential Evolution (DE), Hybrid Harmony Search (HS-BFGS) and Grey Wolf Optimizer (GWO). From among soft computing methods, the fuzzy logic has a special place due to its very appropriate ability and flexibility in modeling uncertainties in linguistic expressions and problems in terms of mathematical relations [7-9]. The risks of Alavian dam construction operations was investigated by Haghshenas et al (2016) using the fuzzy multiple criteria decision making (FMCDM) technique [10]. The protection of the body slope of the reservoir dam in Iran was evaluated by Yousefi Rad et al (2012) and analyzed through Fuzzy analytical hierarchy process [11].

Risk management is one of the issues having complexity and uncertainty in the evaluation and investigation of problems due to the influence of different factors. In the recent decade, with the increase of construction projects, the evaluation and analysis of projects' risks have led to the correct understanding of the process of projects and a significant reduction in financial losses and casualties. There are different methods for the study and management of risk, including the fault tree method, meta-heuristic algorithms, Monte Carlo simulation technique and multi-criteria decision-making methods [12-19]. The application of Monte Carlo simulation for risk assessment [20] was evaluated

\footnotetext{
* Corresponding author: sina.shaffiee@gmail.com
} 
by Yun-Fu (2008). The optical investigations for compartment fire risk analysis were carried out by Siu Kui (2007). They used Monte Carlo simulation as soft computing method in their study [21].

Therefore, in this study, the risk in the tunneling project on the third section of Ghomrud tunnel has been investigated and evaluated using one of the most practical instruments of soft computing, the fuzzy clustering technique. Classification and evaluation of geological units is one of the risk management methods in tunneling projects. Therefore, considering physical and mechanical properties of geology along the project's route, three influential factors in the process of design and execution of project are selected respectively as follows: Overburden $(\mathrm{H})$, internal friction angle $(\phi)$ and cohesion $(\mathrm{C})$. Then, after the required analyses, results are validated with drilling rate index (DRI) obtained through the execution of project. Finally, results show an appropriate match with experimental results, indicating the proper application of fuzzy logic in the risk analysis.

In fact, the goal of geological units' classification is addressing the most important hazards and suitable prediction of project based on the limitation of resources. Therefore, using fuzzy clustering technique based on uncertain systems, a high level of precision in data analysis can be obtained.

\section{Ghomrud Tunnel}

In recent decades, with the increase of population, the need for the growth of construction projects has led to the increase of investments in the infrastructures of Iran. One of the important infrastructures in the area of construction is tunneling projects which have a significant role in the growth of industry, transportation, supply and transfer of water. Therefore, the present research on the third section of Ghomrud tunnel has been conducted as one of the greatest tunneling projects. Evaluation of the project's risk in order to consider the design and execution conditions in tunnel due to its location in Sanandaj-Sirjan zone which is one of the most risky geological zones in Iran has a special place. The tunneling project's route is located in six geological units with nine different geological properties. Figure 1. shows the location of project's execution [22-24].

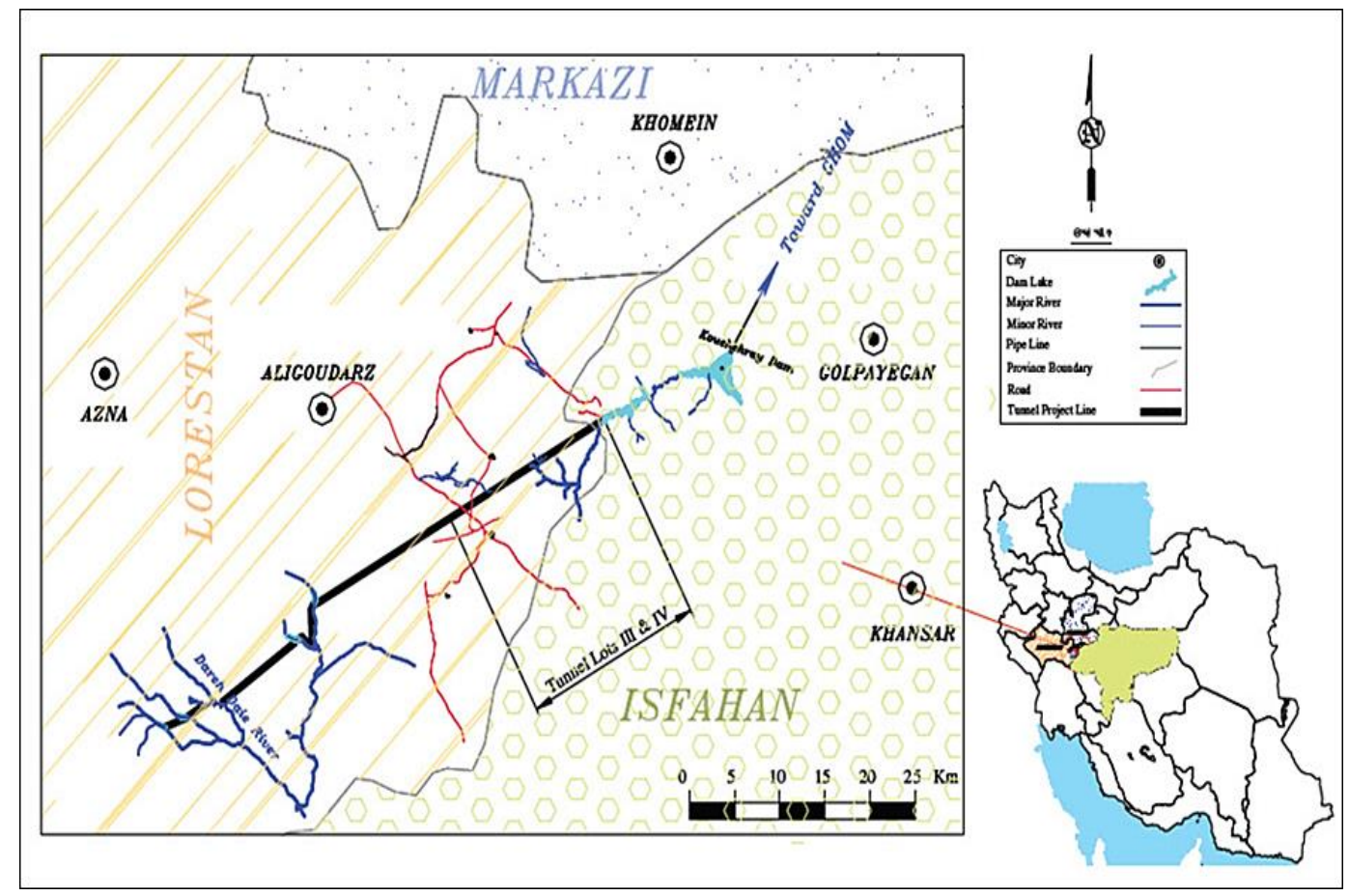

Figure 1. Location of the Tunneling Project [25]

Technical specifications of tunnel include: tunnel length is $36 \mathrm{Km}$ and excavation is done by an EPB-TBM. Excavation diameter, final diameter and excavation volume are $4.69 \mathrm{~m}, 3.8 \mathrm{~m}$ and $195000 \mathrm{~m}^{3}$, respectively [26]. In addition, Table 1. shows physical and mechanical parameters of geological units along the project's route. Considering information in Table 1, there are six geological units with nine different geological properties along the project's route. According to the important role of strength in geological units, the internal friction angle $(\phi)$ and cohesion $(\mathrm{C})$ are involved in geological engineering characteristics classifications. However, Overburden $(\mathrm{H})$ is one of other different effective factors for assessment of geological engineering characteristics in tunneling project, because it has key role in value of loading and tension [27]. 
Table 1. Mechanical Properties and Drilling Rate Index (DRI) [28]

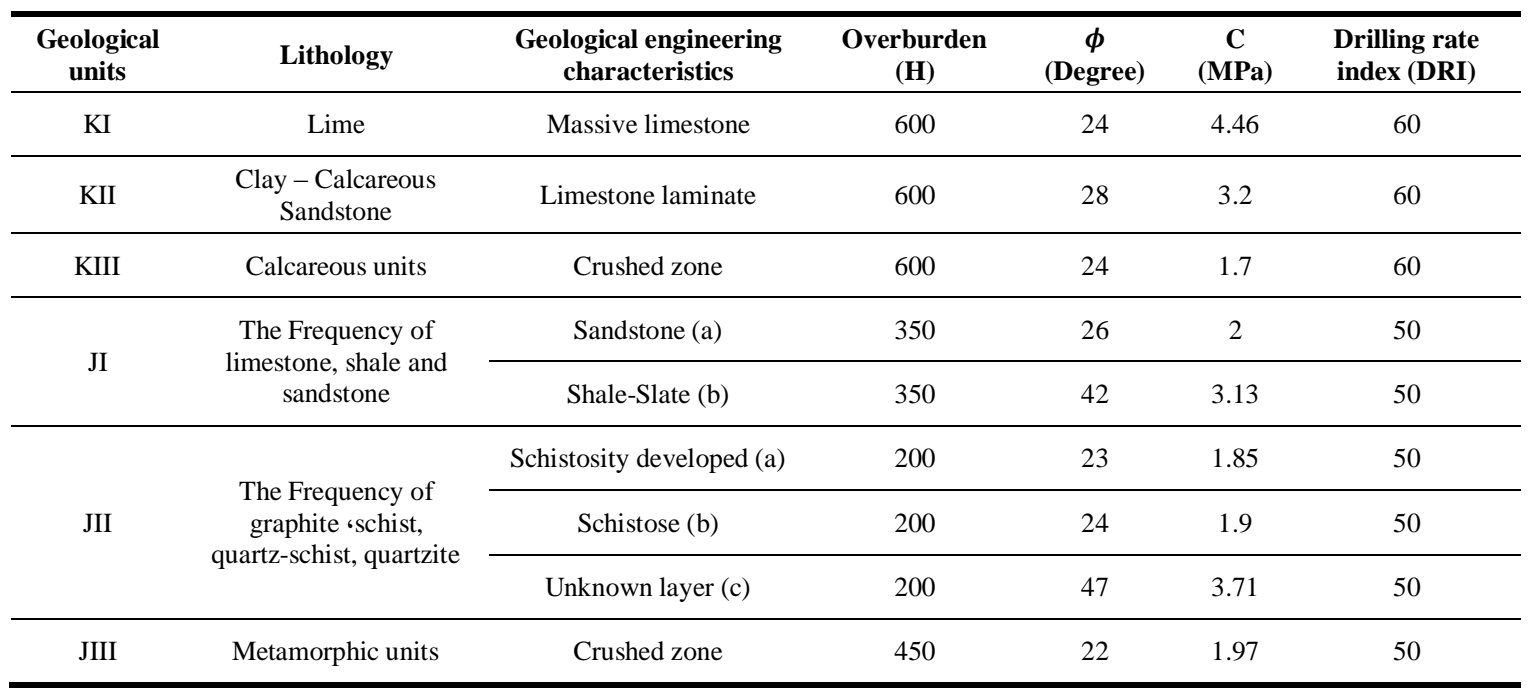

\section{Fuzzy Clustering Technique}

In a paper under the title "theory of fuzzy sets", the basis and foundation of fuzzy logic have been provided by Zadeh in 1965 [29]. The fuzzy logic is a multivalued logic which is used as a powerful and flexible instrument contrary to the classic logic for modeling certainties and uncertainties and linguistic expressions in terms of mathematical relations [30-33]. One of the applications of the fuzzy logic is fuzzy classification. Fuzzy C-means (FCM) method was proposed by Bezdek based on the generalization of Hard C-means (HCM) clustering method [34]. High convergence and accuracy are among advantages of this algorithm. This algorithm has been proposed in 4 steps based on the iterative optimization, including:

In the first step, the number of clusters $\mathrm{c}$ is determined. The numerical value of $\mathrm{c}$ is always larger than or equal to 2 and smaller than or equal to $\mathrm{n}$ (number of samples). After determining the number of clusters (numerical value of c), the value of weighting factor $\mathrm{m}$ ' is determined. The weighting factor determines the amount of fuzziness in the clustering process. $\mathrm{U}^{(0)}$ is guessed as the initial partitioned matrix and the number of each step or iteration of this algorithm is determined with $\mathrm{r}$ value.

Second and Third Steps: in the second step, the center of clusters $\left(V_{i}^{(r)}\right)$ is calculated per iteration. Then, in the next step, using equations (1) to (5), the partitioned matrix for rth iteration is updated as $\left(U^{-(r)}\right)$ [35].

$\mu_{i k}^{(r+1)}=\left[\sum_{j=1}^{c}\left(\frac{d_{i k}^{(r)}}{d_{j k}^{(r)}}\right)^{\frac{2}{m^{\prime}-1}}\right]^{-1}$ for $I_{k}=\phi$

$I_{k}=\left\{i \mid 2 \leq C<n ; d_{i k}^{(r)}=0\right\}$

$\mu_{i k}^{(r+1)}=0$ for all classes $i$ where $i \in \widetilde{I_{k}}$

$\widetilde{I_{k}}=\{1,2, \ldots, c\}-I_{k}$

$\sum_{i \in I_{k}} \mu_{i k}^{(r+1)}=1$

Where, $\mu_{i k}^{(r+1)}$ shows the membership degree of kth data in the ith cluster for $r+1$ iteration of the algorithm. In addition, $d_{i k}$ shows the Euclidean distance between the center of the ith cluster and kth data.

Then, in the final step, the conclusion step of clustering or in other words, the accuracy of algorithm is investigated. If Equation 6. is satisfied, the result of clustering will reach a desired optimization. Under such condition, calculations can be stopped. Otherwise, the second step and its calculations are evaluated. In the following relation, $\epsilon_{L}$ shows the accuracy level of algorithm

$\left\|\widetilde{U}^{(r+1)}-\widetilde{U}^{(r)}\right\|=\epsilon_{L}$ 


\section{Modeling and Discussion}

This study evaluated 9 different kinds of Geological engineering characteristics by laboratory and field studies. In field studies, the drilling rate index (DRI) is measured, while laboratory tests are provided with four important physical and mechanical characteristics of rocks as clustering optimization for risk analysis. In the present study, for the modeling and classification of data in Table 1, first, data are collected and normalized. Then, after the preparation of the pseudo-code of FCM algorithm in MATLAB, the algorithm's control parameters are adjusted in order to have a desired optimization, including:

The weighting parameter of $m^{\prime}=2$, maximum iteration of 100, minimum acceptance precision of $\epsilon_{L}=0.00001$ and the number of clusters $c=2$.

In the next step, the normalized data are called in the algorithm as input data and after the analysis conducted in the 9th step, the algorithm is converged and the condition of minimum acceptance precision is satisfied; therefore, the algorithm's operation is stopped. Classification and investigation of geological units are one of the most important sections in the project management and prediction. Results of evaluation and optimization of data are shown in Tables 2 and 3. Furthermore, Figures 2 and 3. show the process of cost function's optimization in the algorithm and the location of data.

Table 2. Optimization and Classification of Risk by Fuzzy Clustering Technique

\begin{tabular}{|c|c|c|c|c|}
\hline \multirow{2}{*}{$\frac{\text { Geological units }}{\text { KI }}$} & \multicolumn{2}{|c|}{ Optimum partition } & \multicolumn{2}{|c|}{ Classification } \\
\hline & 0.841 & 0.159 & \multirow{4}{*}{ First Class } & $\mathrm{KI}$ \\
\hline KII & 0.981 & 0.019 & & KII \\
\hline KIII & 0.75 & 0.249 & & KIII \\
\hline JIII & 0.518 & 0.482 & & JIII \\
\hline JI (a) & 0.121 & 0.879 & \multirow{5}{*}{ Second Class } & JI (a) \\
\hline JI (b) & 0.371 & 0.629 & & JI (b) \\
\hline JII (a) & 0.086 & 0.915 & & JII (a) \\
\hline JII (b) & 0.073 & 0.927 & & JII (b) \\
\hline JII (c) & 0.325 & 0.675 & & JII (c) \\
\hline
\end{tabular}

Table 3. Distance of Criteria from Centers of Clusters

\begin{tabular}{ccc}
\hline Criteria & First cluster & Second cluster \\
\hline $\mathrm{C}_{1}$ & 0.925 & 0.456 \\
$\mathrm{C}_{2}$ & 0.574 & 0.615 \\
$\mathrm{C}_{3}$ & 0.695 & 0.514 \\
\hline
\end{tabular}

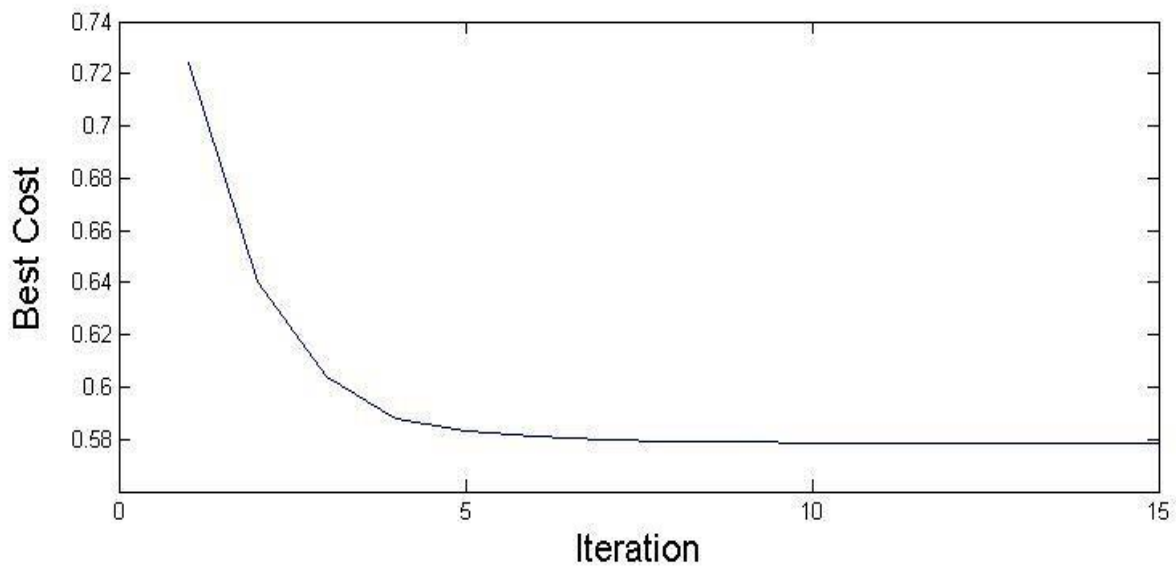

Figure 2. The Best Cost of Optimization Process by FCM 


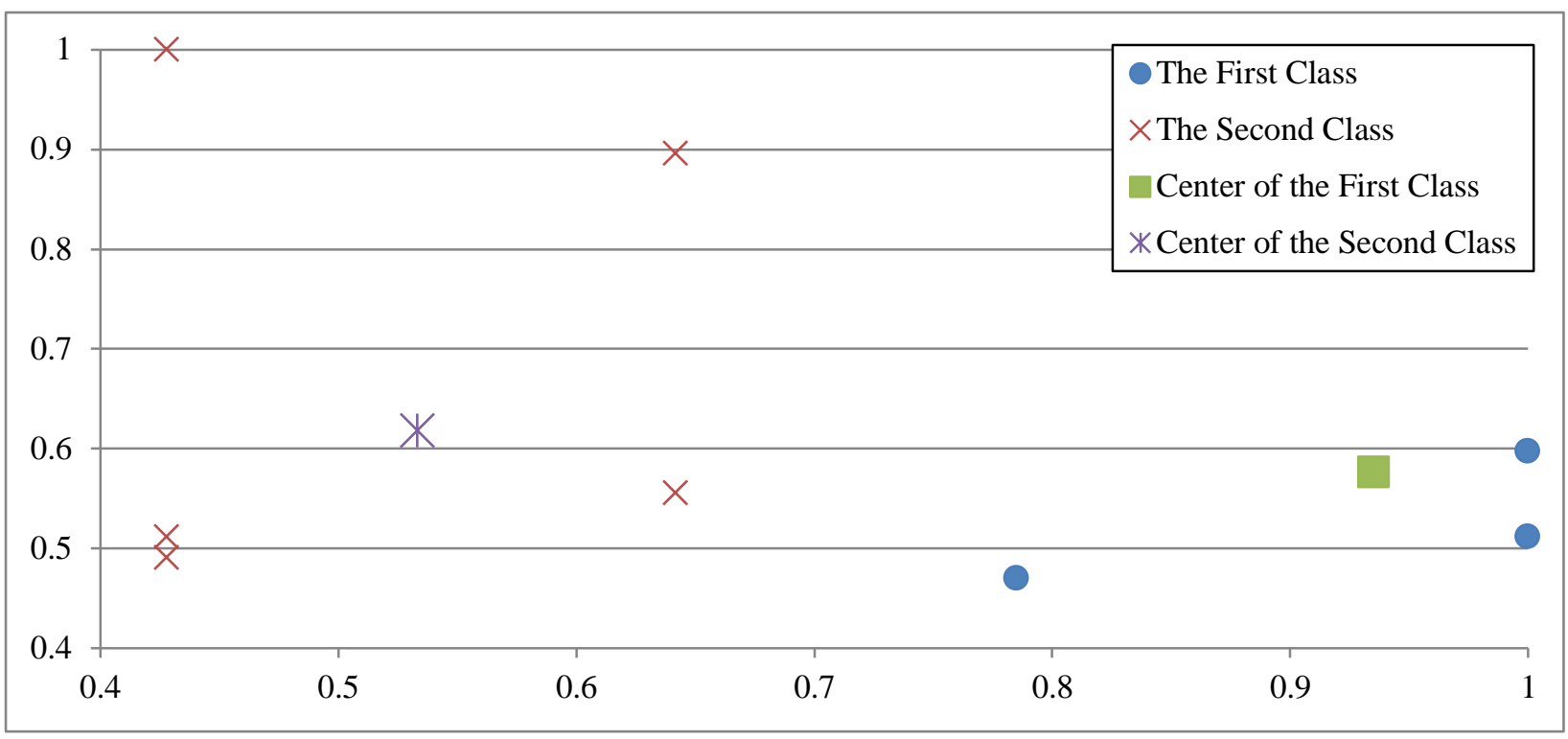

Figure 3. The Positions of Each Data and Centers of Clusters

In each study, validation is an important and fundamental section of research. Therefore, in the present paper, after the evaluation and analysis of data, results are validated with drilling rate index (DRI) based on Table 4. Only in section JIII, this classification is located in a more powerful section opposed to DRI. One of the reasons of the location of this section in another class is the possibility of occurrence of error in experimental measurements, but based on the analysis results, FCM algorithm places this section in the first class and it is accurately validated based on the number of other classifications with DRI. Of course, if error is also due to the algorithm, the algorithm is a desired estimation in the classification of other geological units and the error is highly acceptable. Thus, based on the validation of data, it is determined that FCM technique is a reliable and efficient method for the classification of geological units considering the tested physical and mechanical properties. In addition, based on the analyses conducted, it is determined that the project's route is located in two strong and weak regions in terms of geological risks. In the risk management, risk reducing methods are introduced for inevitable risks. Therefore, considering the inevitability of geological risks in the tunneling project of the third section of Ghomrud tunnel, some suggestions can be provided in order to reduce geological risks, including: the increase of reliability coefficient in the design of tunnel, the increase of the executive team's safety and application of appropriate instruments and facilities with drilling condition in weak zones.

Table 4. Result of Classification by FCM Compared to DRI

\begin{tabular}{lll}
\hline Classification of Geological Units & Drilling Rate Index (DRI) \\
\hline \multirow{3}{*}{ First Class } & KI & 60 \\
\cline { 2 - 3 } & KII & 60 \\
\cline { 2 - 3 } & KIII & 60 \\
\hline \multirow{3}{*}{ Second Class } & JIII & 50 \\
\cline { 2 - 3 } & JI (a) & 50 \\
\cline { 2 - 3 } & JI (b) & 50 \\
\hline & JII (a) & 50 \\
\hline & JII (b) & 50 \\
\hline
\end{tabular}

\section{Conclusion}

In recent decades, concurrent with the increase of vague and uncertain problems, the application of soft computing methods also increased. One of the most important methods of soft computing is the theory of fuzzy sets with the ability of solving large-scale problems with many variables. Furthermore, important problems in risk management 
often have many influential variables and the ability of full match with fuzzy concepts. Therefore, in this paper, using fuzzy clustering technique, geological units of the region in the execution of tunneling project in the third section of Ghomrud tunnel are evaluated. The obtained results are validated with drilling rate index (DRI), indicating the high ability of FCM algorithm in the classification of geological units in tunneling projects and an appropriate provision of the condition of the region for evaluating the risk management in the project.

\section{References}

[1] Bouzon, Marina, Kannan Govindan, Carlos M. Taboada Rodriguez, and Lucila MS Campos. "Identification and analysis of reverse logistics barriers using fuzzy Delphi method and AHP." Resources, Conservation and Recycling 108 (2016): 182-197.

[2] Sabzi, Hamed Zamani, Delbert Humberson, Shalamu Abudu, and James Phillip King. "Optimization of adaptive fuzzy logic controller using novel combined evolutionary algorithms, and its application in Diez Lagos flood controlling system, Southern New Mexico." Expert Systems with Applications 43 (2016): 154-164.

[3] Kuo, R. J., C. H. Mei, F. E. Zulvia, and C. Y. Tsai. "An application of a metaheuristic algorithm-based clustering ensemble method to APP customer segmentation." Neurocomputing 205 (2016): 116-129.

[4] Zhou, Yongquan, Qifang Luo, Jian Xie, and Hongqing Zheng. "A Hybrid Bat Algorithm with Path Relinking for the Capacitated Vehicle Routing Problem." In Metaheuristics and Optimization in Civil Engineering, pp. 255-276. Springer International Publishing, 2016.

[5] Fekrmandi, Hadi, Muhammet Unal, Sebastian Rojas Neva, Ibrahim Nur Tansel, and Dwayne McDaniel. "A novel approach for classification of loads on plate structures using artificial neural networks." Measurement 82 (2016): 37-45.

[6] Yagiz, Saffet, and Halil Karahan. "Application of various optimization techniques and comparison of their performances for predicting TBM penetration rate in rock mass." International Journal of Rock Mechanics and Mining Sciences 80 (2015): 308-315.

[7] Shams, Samira, Masoud Monjezi, Vahid Johari Majd, and Danial Jahed Armaghani. "Application of fuzzy inference system for prediction of rock fragmentation induced by blasting." Arabian Journal of Geosciences 8, no. 12 (2015): 10819-10832.

[8] Haghshenas, Sina Shaffiee, Mostafa Yousefi Rad, and Sami Shaffiee Haghshenas. "Mechanostratigraphy of cretaceous Rocks by Fuzzy Logic in East Arak, Iran. the 4th International Workshop on Computer Science and Engineering - Summer, WCSE, 2014.

[9] Mostafa Yousefi Rad, Sina Shaffiee Haghshenas, and Sami Shaffiee Haghshenas. "Evaluation Rock Mass Deformation Modulus based on Fuzzy Logic."

[10] Haghshenas, Sina Shaffiee, Mir Ahmad Lashteh Neshaei, Pouyan Pourkazem, and Sami Shaffiee Haghshenas. "The Risk Assessment of Dam Construction Projects Using Fuzzy TOPSIS (Case Study: Alavian Earth Dam)." Civil Engineering Journal 2, no. 4 (2016): 158-167.

[11] Rad, Mostafa Yousefi, Sina Shaffiee Haghshenas, Payam Rajabzade Kanafi, and Sami Shaffiee Haghshenas. "Analysis of Protection of Body Slope in the Rockfill Reservoir Dams on the Basis of Fuzzy Logic." In IJCCI, pp. 367-373. 2012.

[12] Bozdag, Erhan, Umut Asan, Ayberk Soyer, and Seyda Serdarasan. "Risk prioritization in Failure Mode and Effects Analysis using interval type-2 fuzzy sets." Expert Systems with Applications 42, no. 8 (2015): 4000-4015.

[13] Li, Shu-cai, Zong-qing Zhou, Li-ping Li, Zhen-hao Xu, Qian-qing Zhang, and Shao-shuai Shi. "Risk assessment of water inrush in karst tunnels based on attribute synthetic evaluation system." Tunnelling and underground space technology 38 (2013): $50-58$.

[14] Vílchez, Juan A., Vicenç Espejo, and Joaquim Casal. "Generic event trees and probabilities for the release of different types of hazardous materials." Journal of Loss Prevention in the Process Industries 24, no. 3 (2011): 281-287.

[15] Fouladgar, Mohammad Majid, Abdolreza Yadani-Chamzini, and M. H. Basiri. "Risk evaluation of tunneling projects by fuzzy Topsis." In International conference on management, no. 1, pp. 1219-1232. 2011.

[16] Farret, Régis, Philippe Gombert, Franz Lahaie, Auxane Cherkaoui, Stéphane Lafortune, and Pierre Roux. "Design of fault trees as a practical method for risk analysis of CCS: application to the different life stages of deep aquifer storage, combining long-term and short-term issues." Energy Procedia 4 (2011): 4193-4198.

[17] Amigun, Bamikole, Daniel Petrie, and Johann Görgens. "Economic risk assessment of advanced process technologies for bioethanol production in South Africa: Monte Carlo analysis." Renewable Energy 36, no. 11 (2011): 3178-3186.

[18] Vaurio, Jussi K. "Ideas and developments in importance measures and fault-tree techniques for reliability and risk analysis." Reliability Engineering \& System Safety 95, no. 2 (2010): 99-107.

[19] Lindhe, Andreas, Lars Rosén, Tommy Norberg, and Olof Bergstedt. "Fault tree analysis for integrated and probabilistic risk analysis of drinking water systems." Water research 43, no. 6 (2009): 1641-1653. 
[20] Wu, Yun-Fu. "Correlated sampling techniques used in Monte Carlo simulation for risk assessment." International Journal of Pressure Vessels and Piping 85, no. 9 (2008): 662-669.

[21] Au, Siu Kui, Zhi-Hua Wang, and Siu-Ming Lo. "Compartment fire risk analysis by advanced Monte Carlo simulation." Engineering Structures 29, no. 9 (2007): 2381-2390.

[22] Jalilvand, Pooria, Sina Shaffiee Haghshenas, Sami Shaffiee Haghshenas, Mehran Honarmand Javan, Iran Astara, Iran Rasht, and Iran Arak. "Evaluation of Dynamic Resistance of the Toyserkan Doolayi Tunnel by Rock Bolt and Reinforced Shotcrete Composite System." In Tunneling and Underground Construction, pp. 376-384. ASCE, 2014.

[23] Jalilvand P, Haghshenas Shaffiee S. "The study stability of Toyserkan Doolayi Tunnel using reinforce shotcrete and rock bolt under static condition. In": The 23rd International Mining Congress and Exhibition of Turkey.2013.

[24] Ahmadi, M., \& Saeidi, O. “ Prediction of TBM Advance Rate using Artificial Neural Networks (Study of third section of Ghomrud Tunnel)”. 8th Iranian Tunnelling Conference. Tehran, Iran.2009.

[25] Farrokh, Ebrahim, and Jamal Rostami. "Correlation of tunnel convergence with TBM operational parameters and chip size in the Ghomroud tunnel, Iran." Tunnelling and Underground Space Technology 23, no. 6 (2008): 700-710.

[26] SCE Company. Report of the third and fourth sections of Ghomrud tunnel.2004.

[27] Javan, M. Honarmand, S. Shaffiee Haghshenas, P. Rajabzadeh Kanafi, and H. O. S. E. I. N. Zartaj. "Investigating The Effect of Soil Behavioral Model On The Performance of Micro Pile System Under Dynamic Loading." In Proceedings of the 4th European Conference of Civil Engineering (ECCIE'13), Antalya, Turkey October, pp. 8-10. 2013.

[28] Ahmadi, M., Shabani, A.,H., Foroughi, M., “ Prediction of TBM Penetration rate in hard rock drilling using NTH model (Case Study of Ghomrud Tunnel)”. Iranian Journal of Mining Engineering (IRJME)1.1 (2006): 33-40.

[29] Zadeh, Lotfi A. "Fuzzy sets." Information and control 8, no. 3 (1965): 338-353.

[30] Mikaeil, Reza, Yilmaz Ozcelik, Reza Yousefi, Mohammad Ataei, and Seyed Mehdi Hosseini. "Ranking the sawability of ornamental stone using Fuzzy Delphi and multi-criteria decision-making techniques." International Journal of Rock Mechanics and Mining Sciences 58 (2013): 118-126.

[31] Mikaeil, Reza, Mohammad Ataei, and Reza Yousefi. Evaluating the Power Consumption in Carbonate Rock Sawing Process by Using FDAHP and TOPSIS Techniques. INTECH Open Access Publisher, 2011.

[32] Cheng, Jao-Hong, and Chih-Huei Tang. "An application of fuzzy Delphi and fuzzy AHP for multi-criteria evaluation on bicycle industry supply chains." WSEAS Transactions on Systems and Control 4, no. 1 (2009): 21-34.

[33] Ertuğrul, İrfan, and Ayşegül Tuş. "Interactive fuzzy linear programming and an application sample at a textile firm." Fuzzy Optimization and Decision Making 6, no. 1 (2007): 29-49.

[34] Bezdek, James C., Robert Ehrlich, and William Full. "FCM: The fuzzy c-means clustering algorithm." Computers \& Geosciences 10, no. 2-3 (1984): 191-203.

[35] Koorepazan Dezfuli, A. "Principles of fuzzy set theory and its applications in the modeling of water engineering problems, Publishing of Collegiate Jahad Uni. of Amirkabir University, Tehran." (2008). 